\title{
EORTC continues to support the appropriate treat- ment of adult patients with G-CSF to prevent febrile neutropenia: guideline updates
}

The European Organisation for Research and Treatment of Cancer (EORTC) has updated its 2006 guideline on the use of granulocyte colony-stimulating factor (G-CSF) for the prevention of febrile neutropenia (FN), a sometimes fatal condition in which a loss of neutrophils in patients receiving chemotherapy leads to infections and fever. The guideline provides recommendations on the assessment of risk factors for FN, and the choice of G-CSF formulation.

Keywords: Febrile neutropenia, G-CSF, leukaemia, lymphoma, myelosuppression, risk

\begin{abstract}
Background
Patients undergoing chemotherapy for cancer are at risk of developing febrile neutropenia (FN), a condition in which a loss of neutrophils leads to infection, fever and sepsis, and is fatal in 9.5 to 12.5 per cent of cases. Patients aged over 65, and those undergoing myelosuppressive therapy appear to be most at risk, and may have their chemotherapy delayed, or treatment doses reduced, to minimize the effects of FN. As a consequence, they are then more prone to treatment failure and poorer clinical outcome with regard to their cancer, particularly solid tumours and lymphoma.
\end{abstract}

Prophylactic treatment is available in the form of G-CSF, which boosts and replenishes the body's supply of neutrophils. This preventive measure reduces hospital admissions, antibiotic use and the need for dose-reduction. Treatment can be with one of several approved forms of recombinant G-CSF, including filgrastim and its biosimilars, or the pegylated version of filgrastim. All three are considered equivalent in clinical efficacy and safety. Their use, however, requires caution, and needs to be limited to patients who are deemed to be most at risk of FN.

To help guide the decision-making process for managing patient treatment and care, EORTC set up a working party in 2005 to systematically review available data and derive evidence-based recommendations on the most appropriate way to use G-CSF in adult patients undergoing chemotherapy. This European Guidelines Working Party published its first set of recommendations in 2006. These guidelines were revised in 2010 following a new systematic review owing to several developments, including the understanding of the factors that predispose patients to the onset of FN, and the availability of new models for assessing risk [1].

\section{Issues considered for the guideline updates}

The updated guideline, which is intended to complement the previously published European Society for Medical Oncology (ESMO) guideline on the use of G-CSF for prevention of chemotherapy-induced FN in patients with cancer, takes into account a range of issues regarding different potential risk factors that affect the likelihood of patients developing FN.

Febrile neutropenia is defined as an absolute neutrophil count of $<0.5 \times 109 / \mathrm{L}$, or $1.0 \times 109 / \mathrm{L}$ predicted to fall below $0.5 \mathrm{x}$ 109/L within 48 hours, with fever or clinical signs of sepsis. Fever is defined as a rise in auxiliary temperature to $>38.5^{\circ} \mathrm{C}$ sustained for at least one hour.
Among the issues considered for the guideline update was the assessment of risk that a patient will develop FN. Risk factors which affect the likelihood of this include the tumour type, chemotherapy regime such as the type, frequency and dosage of chemotherapeutic agents, and patient-related factors such as whether they have experienced FN previously. Various risk indices are available for assessing who is most likely to develop FN, including that produced by the Multinational Association for Supportive Care in Cancer (MASCC) in which a score of 21 or above indicates low risk.

There is consensus that treatment with G-CSF should be given to a patient with solid tumour or lymphoma if their risk goes above a threshold of 20 per cent, according to guidelines from Canada, Europe (EORTC and ESMO) and USA (American Society of Clinical Oncology (ASCO) and the National Comprehensive Cancer Network (NCCN)).

Another issue that clinicians have to contend with is whether or not to give prophylactic antibiotic medication instead of, or in combination with, G-CSF. Caution is generally stressed with regard to antibiotics owing to the need to minimize the development of antibiotic resistant infections. G-CSF may therefore appeal as an alternative way to minimize the risk of infections and fever in patients undergoing chemotherapy, but then clinicians must weigh up the potential adverse effects of G-CSF treatment. These include a small risk that patients may develop secondary cancer, such as myelodysplastic syndrome, acute myeloid leukaemia, or acute lymphoblastic leukaemia.

Updating the guideline involved a new review of literature published between 2006 and July 2009, on studies concerning adults aged 18 and over, with solid tumours or lymphoma, as well as evidence presented at meetings held between April 2006 and December 2009. EORTC also took into account the 2009 NCCN and 2006 ASCO guidelines.

Summary of the 2010 EORTC guideline updates

Recommendation 1: patient-related risk factors for increased incidence of FN and complications of FN

The updated guideline confirms that patients are at increased risk of $\mathrm{FN}$ if the patient:

- is aged 65 years or over

- is at an advanced stage of disease

- has had previous experience of FN 
- has had prior chemotherapy or has intense chemotherapy scheduled.

The recommendations on the process of evaluating risk factors remain in line with previously published guidelines.

\section{Recommendation 2: chemotherapy regimens associated with increased risk of FN}

New targeted agents added to chemotherapy regimens can exacerbate myelosuppression and hence febrile neutropenia, for example, in patients with non-small cell lung cancer given cetuximab or bevacizumab. The updated guideline lists some of the various drug combinations that have a potentially elevated risk of FN and note that, 'Consideration should be given to the elevated risk of FN when using certain chemotherapy regimens.' Furthermore, they stress, 'this list is not comprehensive and there may be other drugs or regimens associated with an increased risk of FN.'

\section{Recommendation 3: G-CSF to support intensive chemo- therapy regimens}

Based on consistent findings in the more recent literature, the updated guideline continues to support the use of prophylactic G-CSF to facilitate the delivery of dose-dense (increased frequency) and dose-intense (increased dose) chemotherapy in an attempt to improve long-term clinical outcomes. This is particularly recommended when more frequent and intensive chemotherapy is likely to have survival benefits.

\section{Recommendation 4: impact of the overall FN risk on G-CSF use}

Recent literature confirms the benefits of G-CSF treatment for preventing $\mathrm{FN}$ in patients with a wide range of malignancies, including breast cancer and lymphoma. Physicians should assess the patient's risk for FN case-by-case in order to make treatment decisions, taking into account patient-related risk factors, the chemotherapy regimen and associated complications and treatment intent. This should be done at the beginning of each treatment cycle. Recent studies confirm that G-CSF has clinical benefits for patients whose FN risk is equal to, or greater than, 20 per cent. The authors note, however, that this guidance is not intended to supersede national guidelines.

\section{Recommendation 5: G-CSF in patients with existing FN}

There are only a limited number of sufficiently powered studies on the effects of G-CSF treatment in patients with an ongoing episode of FN. The EORTC guideline update, in line with ASCO, continues to recommend that 'Treatment with G-CSF for patients with solid tumours and malignant lymphoma and ongoing FN is indicated only in special situations. These are limited to those patients who are not responding to appropriate antibiotic management and who are developing life-threatening infectious complications (such as severe sepsis or septic shock).'

\section{Recommendation 6: choice of formulation}

The updated guideline recommends the use of filgrastim and lenograstim (daily injections) and pegfilgrastim (once per cycle administration) to prevent FN and FN-related complications, where indicated. Filgrastim biosimilars are also included in this recommendation.

In conclusion, the 2010 update of EORTC guideline aims to help optimise local protocols and patient management strategies in hospitals across Europe, in order to improve patient care and clinical outcomes.

\section{Competing interests: None.}

Provenance and peer review: Article prepared based on published scientific or research papers recommended by members of Editorial Board; internally peer reviewed.

\section{Editor's comments}

The EORTC guideline is based on a comprehensive literature review, and concludes that all three G-CSF's are considered therapeutic equivalent choices.

Julie Clayton, PhD, GaBI Journal Editor

\section{Reference}

1. Aapro MS, Bohlius J, Cameron DA, et al. 2010 update of EORTC guidelines for the use of granulocytecolony-stimulating factor to reduce the incidence of chemotherapy-induced febrile neutropenia in adult patients with lymphoproliferative disorders and solid tumours. Eur J Cancer. 47 2011;47(1):8-32. DOI:10.5639/gabij.2013.0201.016

Copyright @ 2013 Pro Pharma Communications International 